\title{
Effect of PDCA Cycle Management Mode on Drug Loss in Inpatient Pharmacy
}

\author{
Xuliang Wu, Jufeng Li, Yanru Luo, Zhidong Zhang* \\ Pharmacy Department, The First Affiliated Hospital of Jinan University, Guangzhou, China \\ Email address: \\ 783287006@qq.com (Xuliang Wu), 1892890348@189.cn (Jufeng Li), 457809436@qq.com (Yanru Luo), \\ 305196618@qq.com (Zhidong Zhang) \\ ${ }^{*}$ Corresponding author
}

\section{To cite this article:}

Xuliang Wu, Jufeng Li, Yanru Luo, Zhidong Zhang. Effect of PDCA Cycle Management Mode on Drug Loss in Inpatient Pharmacy. Journal of Drug Design and Medicinal Chemistry. Vol. 7, No. 1, 2021, pp. 23-26. doi: 10.11648/j.jddmc.20210701.14

Received: February 27, 2021; Accepted: March 20, 2021; Published: March 30, 2021

\begin{abstract}
Objective: To assess effect of PDCA cycle management mode on drug loss in inpatient pharmacy. Methods: From January 2019 to December 2020, we collected the data from hospital work record of inpatient pharmacy each season and data of total drug loss. The valid data of scrap drugs included item name, specification, packing, quantity, wholesale price, expiry date, and scrap reason. In scrap drugs record of hospital, the inpatient pharmacy managers often record drug data from actual situation of inpatient pharmacy and documents from the drug supplier. In addition, we also collected the change of for each season, and compare the result between 2019 and 2020. Result: The results showed that the number of damaged batches reported in 2019 was significantly higher than the number reported in 2020 (122 vs 77), with a difference of $68 \%$ between them. Among the drug loss amount, the loss amount increased with the increase of the number of batches reported to be damaged, and the result of loss amount differed by $54 \%$. In quarter records, we observed that most of the losses occurred in the first quarter and the fourth quarter, with monetary losses of around RMB 2,000 in 2020 and about RMB 3,200 in 2019. Compared with 2019 group, there is a lower amount loss (RMB 10,157.88 vs RMB 5515.14) in the amount loss caused by drug loss in 2020, and the annual reported loss in 2020 group is $54 \%$ of the annual reported loss in 2019. Further, the dollar loss for each quarter in 2020 group was lower than for each quarter in 2019. Conclusion: PDCA cycle management mode effectively reduced drug broken event, that it provided continuous improvement as the inpatient pharmacy carried out this cycle management.
\end{abstract}

Keywords: PDCA Cycle Management, Inpatient Pharmacy, Drug Loss

\section{Introduction}

Drug loss refers to the inventory of drugs stored in the inpatient department pharmacy according to actual needs. These drugs are necessary for clinical first aid and turnover, but the drugs can not be used normally due to various reasons. Such drug losses tend to increase the operating costs of hospital pharmacies [1-3]. Because there are many kinds and quantities of drugs in hospitals, it is difficult for drug administrators to avoid the money loss caused by drug loss [4, 5]. In addition, the Department of Pharmacy is responsible for regular quality inspection of small medicine cabinets and rescue vehicles in the ward, and treatment of drugs with short expiry period and unqualified quality shall be carried out in accordance with relevant regulations $[6,7]$. However, drug loss in inpatient pharmacies has been a hot topic for reducing drug costs. Therefore, managers use different management models to reduce drug loss according to the actual situation of the pharmacy.

In recent years, some reports indicated PDCA cycle management mode can reduced drug loss in inpatient pharmacy $[8,9]$. However, their results were different, that they think different factor improve drug loss. For example, the result of study shown PDCA circulation management mode improve the efficiency of administering drugs so that depletion of drugs due to human error has been reduced [10]. On the other hand, some reports indicated PDCA circulation management mode reduced drug loss by control quantity of drugs purchased so that number of expired drugs has decreased [11, 12]. Therefore, the effect of PDCA circulation management mode for drug loss in inpatient pharmacy is worthy study. The aim of this study was to assess effect of 
PDCA cycle management mode on drug loss in inpatient pharmacy.

\section{Methods}

\subsection{Source of Data and Outcome}

To evaluate the impact of PDCA cycle management mode for drug loss, we designed a retrospective study, and analyzed the valid data. From January 2019 to December 2020, we collected the data from hospital work record of inpatient pharmacy each season and data of total drug loss. The valid data of scrap drugs included item name, specification, packing, quantity, wholesale price, expiry date, and scrap reason. In scrap drugs record of hospital, the inpatient pharmacy managers often record drug data from actual situation of inpatient pharmacy and documents from the drug supplier. In addition, we also collected the change of for each season, and compare the result between 2019 and 2020.

In PDCA cycle management mode, PDCA cycle by the American management master Dr. W. E. first proposed in the 1950s and has been widely used as a scientific comprehensive management method. It consists of four steps: Plan (P), Implement (D), Inspection (C), and Dispose (A), and is one of the basic ways to improve continuous quality [13]. The related measure of inpatient pharmacy included: 1) Specialized nurses are designated to take charge of the collection, storage and cleaning of cardinal drugs; (2) Supplementary drugs should follow the principle of "Take before putting," "In left" and "Out right"; (3) For less used and slow turnover base drugs, apply for reduction of base; (4) shorten the quality inspection time; (5) Unified base drug replacement process. In present study, PDCA cycle management mode only control drug broke event in inpatient pharmacy.

\subsection{Data Analyses}

The data of scrap drugs are presented as numbers and percentages. To identify statistically significant and significantly different, the statistical significance was observed at level of 0.05 . Also, we used mean and standard deviation (SD) to describe result, and establish a database and analyzed the valid data by Excel and SPSS22.0. The valid data included item name, specification, packing, quantity, wholesale price, expiry date, and scrap reason.

\section{Result}

This study was carry out in 2020 and 2019, we assess the effect of PDCA cycle management mode by comparison between drug overdue cases and drug broken of 2020 and 2019. In Figure 1, it displays a cycle PDCA steps for drug loss in inpatient pharmacy. The cycle PDCA steps had 8 steps to improved drug loss in this study, that it includes the following as: (1) Identify and prioritize improvement opportunities of drugs loss; (2) Describe and collect data on the drugs loss; (3) Identify all possible causes of drugs loss; (4) Develop an action plan to reduce drugs loss event; (5) Execution; (6) Check/Study; (7) Standardization of drugs loss prevention; (8) Conclusion \& record. These steps are cyclic with the work cycle.

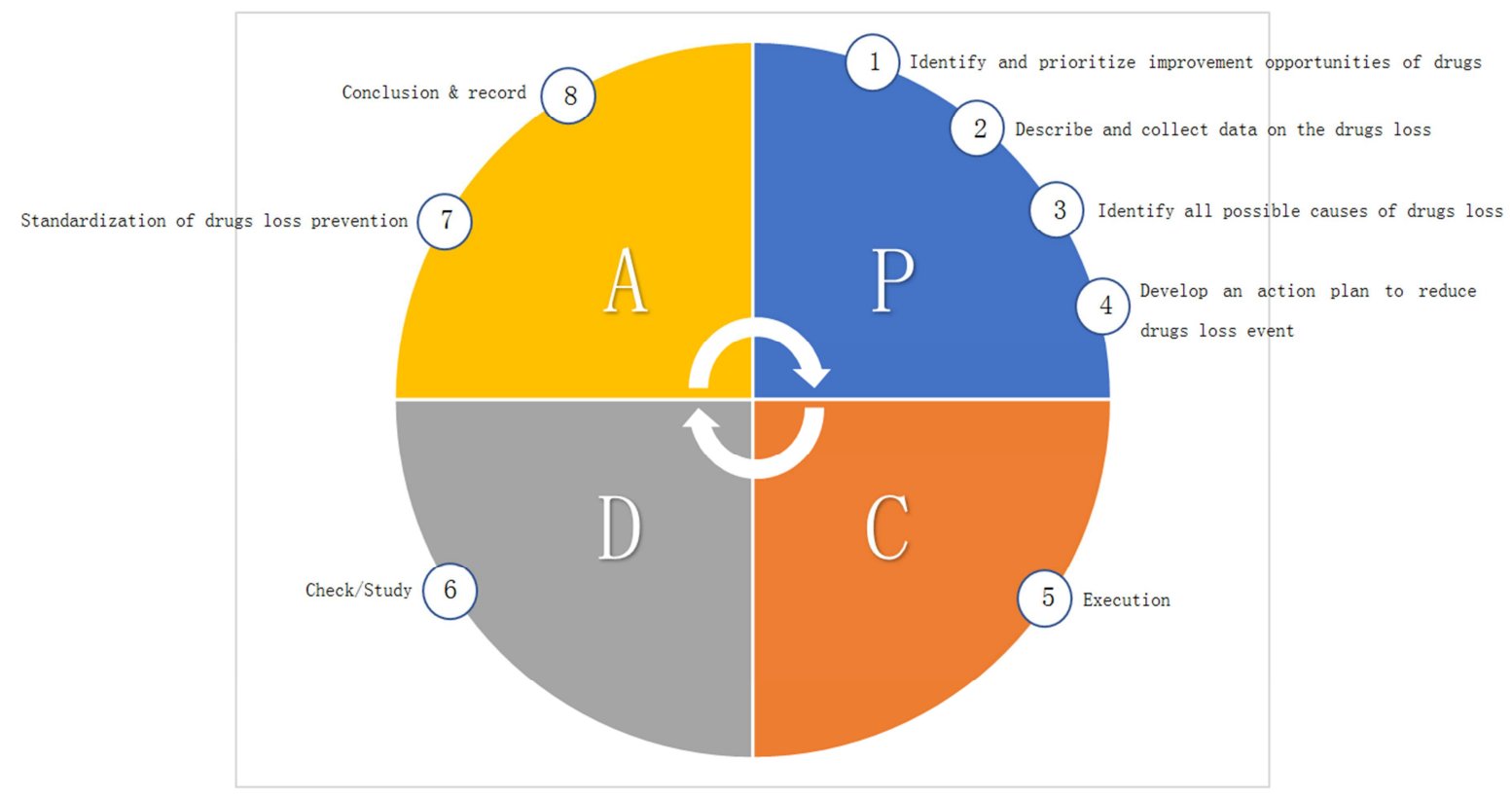

Figure 1. Cycle PDCA steps.

To analyze the causes of drug loss, we collected 2019 and 2020 batch statistics cases from inpatient pharmacy drug loss records. The results showed that the number of damaged batches reported in 2019 was significantly higher than the number reported in 2020 (122 vs 77), with a difference of $68 \%$ between them. Among the drug loss amount, the loss amount increased with the increase of the number of batches reported to be damaged, and the result of loss amount differed by $54 \%$, 
as shown in Table 1.

Table 1. Drug loss reason statistics.

\begin{tabular}{lll}
\hline & Reported loss batches (times) & Amount of drug loss (RMB) \\
\hline 2019 & 122 & 10157.88 \\
2020 & 77 & 5515.14 \\
Difference between two years (\%) & $68 \%$ & $54 \%$ \\
\hline
\end{tabular}

Figure 2 shows the quarterly results for the loss amounts in 2019 and 2020. We observed that most of the losses occurred in the first quarter and the fourth quarter, with monetary losses of around RMB 2,000 in 2020 and about RMB 3,200 in 2019. Compared with 2019 group, there is a lower amount loss
(RMB 10,157.88 vs RMB 5515.14) in the amount loss caused by drug loss in 2020, and the annual reported loss in 2020 group is $54 \%$ of the annual reported loss in 2019 . Further, the dollar loss for each quarter in 2020 group was lower than for each quarter in 2019.

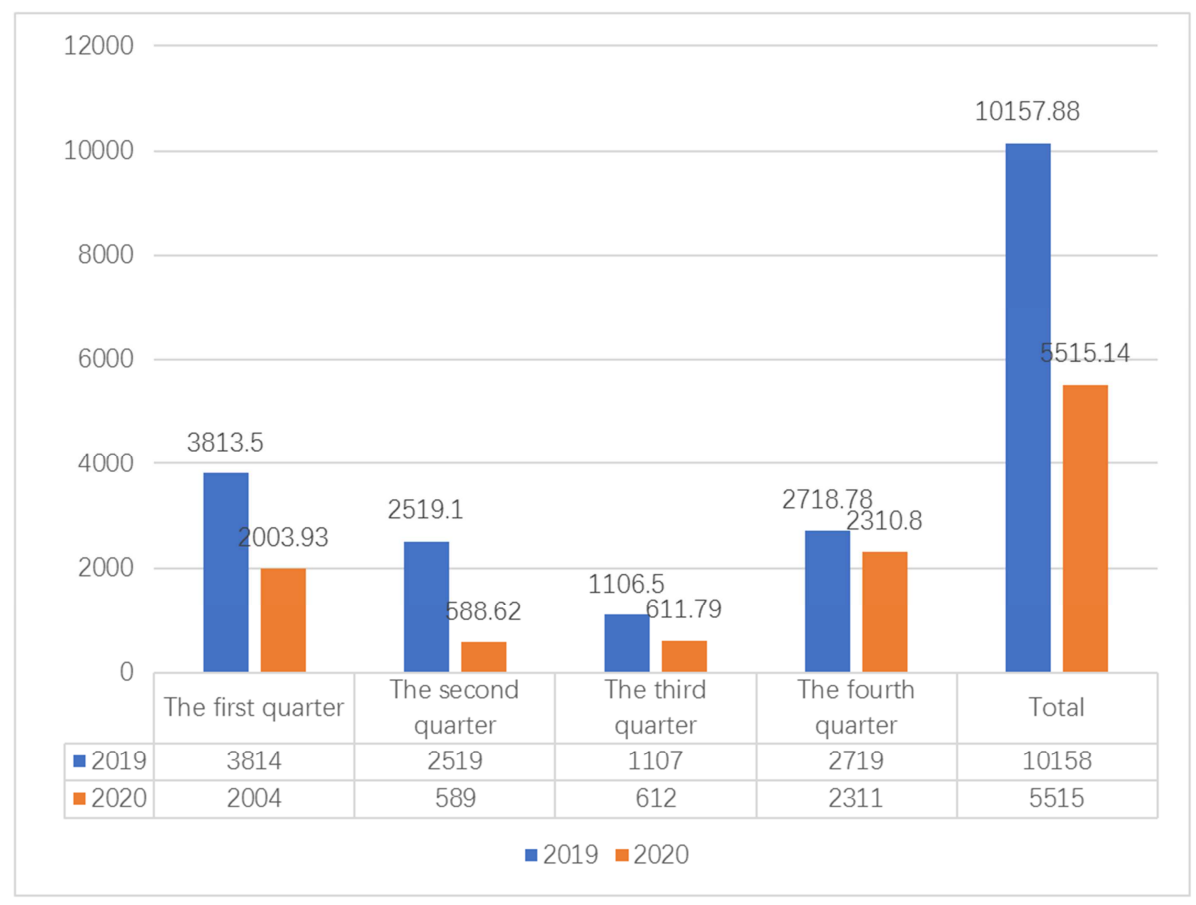

Figure 2. The amount of money loss and number of drug loss in each quarter.

\section{Discussion}

To explore the effect of PDCA cycle management mode on drug loss in inpatient pharmacy, we summarize the result of drug loss and money loss by collecting related data from records of inpatient pharmacy and comparison of drug loss and money loss in 2020 and 2019. In this study, we demonstrated that most money loss and drug loss were made from drug overdue, and we observed most drug loss cases were happen in the fourth quarter of a year. By comparing the loss amount in 2019 and 2020, the results show that the PDCA cycle management model effectively reduces the monetary loss caused by drug loss. However, inpatient pharmacies still need to control the shelf life of drugs. By adjusting the quantity of drugs purchased by inpatient pharmacies, the loss of drugs and money loss can be effectively reduced.

As shown in Table 1, The number of damaged batches reported in 2019 is much higher than the number reported in 2020. This result indicates that PDCA cycle management mode reduces the number of drug losses, and this result is also similar to some reports in related fields [2, 4]. As shown in Figure 2, We found that the total and quarterly monetary losses in 2020 were lower than the total and quarterly losses in 2019, and that the PDCA cycle management model improved the overall monetary losses in inpatient pharmacies due to drug losses. The possible reason is that the PDCA cycle management model adjusts the inventory and circulation cycle of the pharmacy in 2020 according to the loss situation in 2019 , so as to reduce the loss caused by drug expiration. Some reports have also shown similar results, and controlling drug inventory and circulation cycle can change the loss caused by drug expiration $[1,2,6]$. In addition, the PDCA cycle management model also changes the flow of access to drugs so as to reduce drug breakage events. In addition, the most drug loss incidents occurred in the fourth quarter, and the most amount reported occurred in the first and fourth quarters. The reason may be the poor effect of PDCA cycle management mode in the early stage, which leads to the large amount loss in inpatient pharmacy even when there is only a small amount of drug loss. In addition, the PDCA cycle management model had a strong effect in the last step, so inpatient pharmacies 
reported similar losses in the fourth quarter as in the first quarter under the conditions of different drug losses.

several studies in related fields clearly demonstrated that PDCA cycle management had effect to improve drug losses in the hospital sector continuously, even the more cycles were carried out so that its effect is better [14-16]. Likewise, this study indicated PDCA cycle management reduced incidence of drug broken, and it had a better effect as the step progresses.

In limitation, our subjects were drug broken event in inpatient pharmacy, so it is not known whether our results are application to other department or other subjects. Also, because we examined cases from only 4 quarters of a year, the result of this study may have been due to chance. The results must be confirmed in a large study.

Therefore, PDCA cycle management mode may be a useful tool for saving the operation cost of the hospital department in long-term. In addition, it also provides continuous improvement as the department of hospital carry out PDCA cycle management.

In conclusion, PDCA cycle management mode effectively reduced drug broken event, that it provided continuous improvement as the inpatient pharmacy carried out this cycle management.

\section{References}

[1] Wen Y. Application effect of PDCA cycle method in high risk pharmacy management of outpatient pharmacy. Chinese Journal of Health and Nutrition. 2020; 30 (20): 317.

[2] Huang J, Wang JP, Han XN, et al. Application effect of PDCA cycle method in drug expiry management of inpatient pharmacy in our hospital. Journal of Clinical Research \& Practice. 2020; 5 (21): 184-187.

[3] $\mathrm{Xu}$ YH. Application of PDCA cycle method in improving pharmacy management quality and reducing drug application error rate. Chinese Journal of Health and Nutrition. 2020; 30 (17): 384-385.

[4] Zhou YW. Application of PDCA cycle method in near effect pharmacy management of hospital outpatient western medicine pharmac. Chinese Journal of Health and Nutrition. 2020; 30 (17): 394-395.
[5] Li S, Sheng XY, Liang YW, et al. Discussion on the management practice and intervention measures of key monitored drugs in our hospital. China Pharmacy. 2018, 29 (22): 10-15.

[6] Bian GX. Analysis of Clinical Significance of Strengthening Drug Returning Management in Hospital Pharmacy to Ensure Drug Use Safety. Chinese Journal of Pharmacology and Clinics. 2018; 18 (8): 148 149.

[7] Hong XF, Xu YM, Wu XR, Zhang JF. Analysis on the Application Effect of PDCA Cycle in the Normative Management of Outpatient Anesthesia Prescription. Modern Hospital. 2021; 21 (03): 389-391.

[8] Wu ZH, Huang JW. Application of PDCA Cycle in the Management of Automatic Picking Machine. Modern Hospital. 2021; 21 (03): 392-394+398.

[9] Zhao LM, Yan YZ, Lin J, Zhang J, Zhang D, Li T, Zhao JX, Shi QH. Application of PDCA Cycle in Improving the Ethical Review of Clinical Scientific Research Projects. Chinese Journal of Medical Ethics. 2021; 34 (03): 328-332.

[10] Baker-Schena L. Expensive drugs. Eyenet Magazine. 2017; 21 (6): 39-44.

[11] Alqenae A, Steinke D, Keers RN. Prevalence and nature of medication errors and medication-related harm following discharge from hospital to community settings: a systematic review, Drug Saf. 2020; 43: 517-537.

[12] Bonaudo M, Martorana M, Dimonte V, Alfonso AD, Fornero G, Politano G, Gianino MM. Medication discrepancies across multiple care transitions: a retrospective longitudinal cohort study in Italy, PLoS One. 2018; 13: 1-13.

[13] Schroeder P. Using the PDCA cycle. Nursing Quality Connection. 1993; 2 (1): 1, 8.

[14] Liu Y, Luo J, Zhou B C. Application of PDCA cycle method and human machine material loop method in rational use of second class psychotropic drugs. Modern Medicine and Health. 2021; 37 (05): 886-888.

[15] Tang RJ, Huang WJ. The promotion effect of PDCA cycle management intervention on rational application of clinical Chinese patent medicine. Prescription Drug in China. 2021; 19 (03): 54-55.

[16] Liang C L. Effect observation of PDCA cycle method in improving pharmacy management quality [J]. Journal of Mathematical Medicine and Pharmacy. 2021; 34 (03): 461-462. 